George A. Anastassiou (Memphis, TN)

IOANNIS K. Argyros (Lawton, OK)

\title{
ON THE CONVERGENCE OF SECANT-LIKE ALGORITHMS WITH APPLICATIONS TO GENERALIZED FRACTIONAL CALCULUS
}

Abstract. We present local and semilocal convergence results for secantlike algorithms in order to approximate a locally unique solution of a nonlinear equation in a Banach space setting. In the last part of the study we present some choices of the operators involved in fractional calculus where the operators satisfy the convergence conditions.

1. Introduction. In this study we are concerned with the problem of approximating a locally unique solution $x^{*}$ of the nonlinear equation

$$
F(x)=0,
$$

where $F$ is a continuous operator defined on a subset $D$ of a Banach space $X$ with values in a Banach space $Y$.

A lot of problems in computational sciences and other disciplines can be brought into a form like (1.1) using mathematical modelling [7, 11, 15]. The solutions of such equations can be found in closed form only in special cases. That is why most solution methods for these equations are iterative. Iterative methods are usually studied based on semilocal and local convergence. The semilocal convergence analysis is, based on the information around the initial point, to give hypotheses ensuring the convergence of the iterative algorithm; while the local convergence analysis is, based on the information around a solution, to find estimates of the radii of convergence balls as well as error bounds on the distances involved.

2010 Mathematics Subject Classification: 65G99, 65H10, 26A33, 47J25, 47J05.

Key words and phrases: secant-like algorithms, Banach space, semilocal-local convergence, fractional calculus, generalized fractional derivatives.

Received 18 August 2015.

Published online 10 December 2015. 
We introduce the secant-type method defined for $n=0,1,2, \ldots$ by

$$
x_{n+1}=x_{n}-A(F)\left(x_{n}, x_{n-1}\right)^{-1} F\left(x_{n}\right),
$$

where $x_{-1}, x_{0} \in D$ are initial points and $A(F)(x, y) \in L(X, Y)$, the space of bounded linear operators from $X$ into $Y$. There is a plethora of local as well as semilocal convergence theorems for method $(1.2)$ provided that the operator $A$ is an approximation to the Fréchet derivative $F^{\prime}[1,2,5-15]$. In the present study we do not assume that the operator $A$ is related to $F^{\prime}$. This way we expand the applicability of the iterative algorithm (1.2). Notice that many well known methods are special cases of method (1.2).

Newton's method: Choose $A(F)(x, x)=F^{\prime}(x)$ for each $x \in D$.

Secant method: Choose $A(F)(x, y)=[x, y ; F]$, where $[x, y ; F]$ denotes a divided difference of order one [7, 11, 14].

The so-called Newton-like algorithms and many other methods are special cases of method 1.2.

The paper is organized as follows. The semilocal as well as local convergence analysis of method 1.2 is given in Section 2. Some applications to fractional calculus are given in Section 3.

2. Convergence analysis. We present the main semilocal convergence result for method 1.2 .

Theorem 2.1. Let $F: D \subset X \rightarrow Y$ be a continuous operator and let $A(F)(x, y) \in L(X, Y)$. Suppose that there exist $x_{-1}, x_{0} \in D, \eta \geq 0, p \geq 1$, and a function $\varphi:[0, \infty)^{2} \rightarrow[0, \infty)$ continuous and nondecreasing (in each variable separately) such that for all $x, y, z \in D$,

$$
\begin{gathered}
A(F)(z, y)^{-1} \in L(Y, X), \\
\max \left\{\left\|x_{-1}-x_{0}\right\|,\left\|A(F)\left(x_{0}, x_{-1}\right)^{-1} F\left(x_{0}\right)\right\|\right\} \leq \eta, \\
\left\|A(F)(z, y)^{-1}(F(z)-F(y)-A(F)(y, x)(z-y))\right\| \\
\leq \varphi(\|z-y\|,\|y-x\|)\|z-y\|^{p+1}, \\
q:=\varphi(\eta, \eta) \eta^{p}<1
\end{gathered}
$$

and

$$
\bar{U}\left(x_{0}, r\right) \subseteq D,
$$

where

$$
r=\frac{\eta}{1-q} .
$$

Then the sequence $\left\{x_{n}\right\}$ generated by method (1.2) is well defined, remains in $\bar{U}\left(x_{0}, r\right)$ for all $n=0,1,2, \ldots$ and converges to some $x^{*} \in \bar{U}\left(x_{0}, r\right)$ such 
that

$$
\begin{aligned}
\left\|x_{n+1}-x_{n}\right\| & \leq \varphi\left(\left\|x_{n}-x_{n-1}\right\|,\left\|x_{n-1}-x_{n-2}\right\|\right)\left\|x_{n}-x_{n-1}\right\|^{p+1} \\
& \leq q\left\|x_{n}-x_{n-1}\right\|
\end{aligned}
$$

and

$$
\left\|x_{n}-x^{*}\right\| \leq \frac{q^{n} \eta}{1-q} .
$$

Proof. The iterate $x_{1}$ is well defined by method (1.2) for $n=0$ and 2.1. By 2.2 and 2.6 we also have $\left\|x_{1}-x_{0}\right\|=\left\|A(F)\left(x_{0}, x_{-1}\right)^{-1} F\left(x_{0}\right)\right\|$ $\leq \eta<r$, so $x_{1} \in \bar{U}\left(x_{0}, r\right)$ and $x_{2}$ is well defined (by 2.5). Using 2.3 and 2.4 we get

$$
\begin{aligned}
\left\|x_{2}-x_{1}\right\| & =\left\|A(F)\left(x_{1}, x_{0}\right)^{-1}\left[F\left(x_{1}\right)-F\left(x_{0}\right)-A(F)\left(x_{0}, x_{-1}\right)\left(x_{1}-x_{0}\right)\right]\right\| \\
& \leq \varphi\left(\left\|x_{1}-x_{0}\right\|,\left\|x_{0}-x_{-1}\right\|\right)\left\|x_{1}-x_{0}\right\|^{p+1} \leq q\left\|x_{1}-x_{0}\right\|,
\end{aligned}
$$

which shows 2.7 for $n=1$. Then

$$
\begin{aligned}
\left\|x_{2}-x_{0}\right\| & \leq\left\|x_{2}-x_{1}\right\|+\left\|x_{1}-x_{0}\right\| \leq q\left\|x_{1}-x_{0}\right\|+\left\|x_{1}-x_{0}\right\| \\
& =(1+q)\left\|x_{1}-x_{0}\right\| \leq \frac{1-q^{2}}{1-q} \eta<r
\end{aligned}
$$

so $x_{2} \in \bar{U}\left(x_{0}, r\right)$ and $x_{3}$ is well defined.

Assuming $\left\|x_{k+1}-x_{k}\right\| \leq q\left\|x_{k}-x_{k-1}\right\|$ and $x_{k+1} \in \bar{U}\left(x_{0}, r\right)$ for $k=$ $1, \ldots, n$ we get

$$
\begin{aligned}
& \left\|x_{k+2}-x_{k+1}\right\| \\
& \quad=\left\|A(F)\left(x_{k+1}, x_{k}\right)^{-1}\left[F\left(x_{k+1}\right)-F\left(x_{k}\right)-A(F)\left(x_{k}, x_{k-1}\right)\left(x_{k+1}-x_{k}\right)\right]\right\| \\
& \quad \leq \varphi\left(\left\|x_{k+1}-x_{k}\right\|,\left\|x_{k}-x_{k-1}\right\|\right)\left\|x_{k+1}-x_{k}\right\|^{p+1} \\
& \quad \leq \varphi\left(\left\|x_{1}-x_{0}\right\|,\left\|x_{0}-x_{-1}\right\|\right)\left\|x_{1}-x_{0}\right\|^{p}\left\|x_{k+1}-x_{k}\right\| \leq q\left\|x_{k+1}-x_{k}\right\|
\end{aligned}
$$

and

$$
\begin{aligned}
\left\|x_{k+2}-x_{0}\right\| & \leq\left\|x_{k+2}-x_{k+1}\right\|+\left\|x_{k+1}-x_{k}\right\|+\cdots+\left\|x_{1}-x_{0}\right\| \\
& \leq\left(q^{k+1}+q^{k}+\cdots+1\right)\left\|x_{1}-x_{0}\right\| \\
& \leq \frac{1-q^{k+2}}{1-q}\left\|x_{1}-x_{0}\right\|<\frac{\eta}{1-q}=r
\end{aligned}
$$

which completes the induction for 2.7 and shows $x_{k+2} \in \bar{U}\left(x_{0}, r\right)$. We also have, for $m \geq 0$,

$$
\begin{aligned}
\left\|x_{n+m}-x_{n}\right\| & \leq\left\|x_{n+m}-x_{n+m-1}\right\|+\cdots+\left\|x_{n+1}-x_{n}\right\| \\
& \leq\left(q^{m-1}+q^{m-2}+\cdots+1\right)\left\|x_{n+1}-x_{n}\right\| \\
& \leq \frac{1-q^{m}}{1-q} q^{n}\left\|x_{1}-x_{0}\right\| .
\end{aligned}
$$


It follows that $\left\{x_{n}\right\}$ is a Cauchy sequence in the Banach space $X$ and as such it converges to some $x^{*} \in \bar{U}\left(x_{0}, r\right)$. By letting $m \rightarrow \infty$, we obtain 2.8.

Stronger hypotheses are needed to show that $x^{*}$ is a solution of $F(x)=0$.

Proposition 2.2. Let $F: D \subset X \rightarrow Y$ be a continuous operator and let $A(F)(x, y) \in L(X, Y)$. Suppose that there exist $x_{-1}, x_{0} \in D, \eta \geq 0, p \geq 1$, $\mu>0$, and a function $\varphi_{1}:[0, \infty)^{2} \rightarrow[0, \infty)$ continuous and nondecreasing such that for all $x, y \in D$,

$$
\begin{gathered}
A(F)(x, y)^{-1} \in L(Y, X), \quad\left\|A(F)(x, y)^{-1}\right\| \leq \mu, \\
\max \left\{\left\|x_{-1}-x_{0}\right\|,\left\|A(F)\left(x_{0}, x_{-1}\right)^{-1} F\left(x_{0}\right)\right\|\right\} \leq \eta, \\
\|F(z)-F(y)-A(F)(y, x)(z-y)\| \\
\leq \frac{\varphi_{1}(\|z-y\|,\|x-y\|)}{\mu}\|z-y\|^{p+1}, \\
q_{1}:=\varphi_{1}(\eta, \eta) \eta^{p}<1
\end{gathered}
$$

and

$$
\bar{U}\left(x_{0}, r_{1}\right) \subseteq D, \quad \text { where } \quad r_{1}=\frac{\eta}{1-q_{1}} .
$$

Then, the conclusions of Theorem 2.1 for the sequence $\left\{x_{n}\right\}$ hold with $\varphi_{1}$, $q_{1}, r_{1}$ replacing $\varphi, q, r$, respectively. Moreover, $x^{*}$ is a solution of $F(x)=0$.

Proof. Notice that

$$
\begin{aligned}
& \left\|A(F)\left(x_{n}, x_{n-1}\right)^{-1}\left[F\left(x_{n}\right)-F\left(x_{n-1}\right)-A(F)\left(x_{n-1}, x_{n-2}\right)\left(x_{n}-x_{n-1}\right)\right]\right\| \\
& \quad \leq\left\|A(F)\left(x_{n}, x_{n-1}\right)^{-1}\right\|\left\|F\left(x_{n}\right)-F\left(x_{n-1}\right)-A(F)\left(x_{n-1}, x_{n-2}\right)\left(x_{n}-x_{n-1}\right)\right\| \\
& \quad \leq \varphi_{1}\left(\left\|x_{n}-x_{n-1}\right\|,\left\|x_{n-1}-x_{n-2}\right\|\right)\left\|x_{n}-x_{n-1}\right\|^{p+1} \leq q_{1}\left\|x_{n}-x_{n-1}\right\| .
\end{aligned}
$$

Therefore, the proof of Theorem 2.1 can apply. Then, in view of the estimate

$$
\begin{aligned}
\left\|F\left(x_{n}\right)\right\| & =\left\|F\left(x_{n}\right)-F\left(x_{n-1}\right)-A(F)\left(x_{n-1}, x_{n-2}\right)\left(x_{n}-x_{n-1}\right)\right\| \\
& \leq \frac{\varphi_{1}\left(\left\|x_{n}-x_{n-1}\right\|\right)}{\mu}\left\|x_{n}-x_{n-1}\right\|^{p+1} \leq q_{1}\left\|x_{n}-x_{n-1}\right\|,
\end{aligned}
$$

we deduce by letting $n \rightarrow \infty$ that $F\left(x^{*}\right)=0$.

Concerning the uniqueness of the solution $x^{*}$ we have the following result:

Proposition 2.3. Under the hypotheses of Proposition 2.2, suppose moreover that there exists $\varphi_{2}:[0, \infty)^{2} \rightarrow[0, \infty)$ continuous and nondecreasing such that

$$
\|F(z)-F(x)-A(F)(z, y)(z-x)\| \leq \frac{\varphi_{2}(\|z-x\|,\|y-x\|)}{\mu}\|z-x\|^{p+1}
$$

and

$$
\varphi_{2}\left(r_{1}, \eta+r_{1}\right) r_{1}^{p}<1
$$

Then $x^{*}$ is the only solution of $F(x)=0$ in $\bar{U}\left(x_{0}, r_{1}\right)$. 
Proof. The existence of a solution $x^{*} \in \bar{U}\left(x_{0}, r_{1}\right)$ has been established in Proposition 2.2. Let $y^{*} \in \bar{U}\left(x_{0}, r_{1}\right)$ with $F\left(y^{*}\right)=0$. Then

$$
\begin{aligned}
\| x_{n+1}- & y^{*}\|=\| x_{n}-y^{*}-A(F)\left(x_{n}, x_{n-1}\right)^{-1} F\left(x_{n}\right) \| \\
& =\left\|A(F)\left(x_{n}, x_{n-1}\right)^{-1}\left[A(F)\left(x_{n}, x_{n-1}\right)\left(x_{n}-y^{*}\right)-F\left(x_{n}\right)+F\left(y^{*}\right)\right]\right\| \\
& \leq\left\|A(F)\left(x_{n}, x_{n-1}\right)^{-1}\right\|\left\|F\left(y^{*}\right)-F\left(x_{n}\right)-A(F)\left(x_{n}, x_{n-1}\right)\left(y^{*}-x_{n}\right)\right\| \\
& \leq \mu \frac{\varphi_{1}\left(\left\|x_{n}-y^{*}\right\|,\left\|x_{n-1}-y^{*}\right\|\right)}{\mu}\left\|x_{n}-y^{*}\right\|^{p+1} \\
& \leq \varphi_{2}\left(r_{1}, \eta+r_{1}\right) r_{1}^{p}\left\|x_{n}-x^{*}\right\|<\left\|x_{n}-y^{*}\right\|,
\end{aligned}
$$

so we deduce that $\lim _{n \rightarrow \infty} x_{n}=y^{*}$. But since $\lim _{n \rightarrow \infty} x_{n}=x^{*}$, we conclude that $x^{*}=y^{*}$.

Next, we present a local convergence analysis for algorithm (1.2).

Proposition 2.4. Let $F: D \subset X \rightarrow Y$ be a continuous operator and let $A(F)(y, x) \in L(X, Y)$. Suppose that there exist $x^{*} \in D, p \geq 1$, and a function $\varphi_{3}:[0, \infty)^{2} \rightarrow[0, \infty)$ continuous and nondecreasing such that for all $x, y \in D$,

$$
\begin{gathered}
F\left(x^{*}\right)=0, \quad A(F)(y, x)^{-1} \in L(Y, X), \\
\| A(F)(y, x)^{-1}[F(x)- \\
\left.\quad F\left(x^{*}\right)-A(F)(y, x)\left(x-x^{*}\right)\right] \| \\
\leq \varphi_{3}\left(\left\|y-x^{*}\right\|,\left\|x-x^{*}\right\|\right)\left\|y-x^{*}\right\|^{p+1},
\end{gathered}
$$

and

$$
\bar{U}\left(x^{*}, r_{2}\right) \subseteq D,
$$

where $r_{2}$ is the smallest positive solution of the equation

$$
h(t):=\varphi_{3}(t, t) t^{p}-1=0 .
$$

Then the sequence $\left\{x_{n}\right\}$ generated by method (1.2) for $x_{-1}, x_{0} \in U\left(x^{*}, r_{2}\right)-$ $\left\{x^{*}\right\}$ is well defined, remains in $U\left(x^{*}, r_{2}\right)$ for $n=0,1,2, \ldots$ and converges to $x^{*}$. Moreover,

$$
\left\|x_{n+1}-x^{*}\right\| \leq \varphi_{2}\left(\left\|x_{n}-x^{*}\right\|,\left\|x_{n-1}-x^{*}\right\|\right)\left\|x_{n}-x^{*}\right\|^{p+1}<\left\|x_{n}-x^{*}\right\|<r_{2} .
$$

Proof. We have $h(0)=-1<0$ and $h(t) \rightarrow \infty$ as $t \rightarrow \infty$. Then the intermediate value theorem shows that $h$ has positive zeros. Denote by $r_{2}$ the smallest such zero. By hypothesis $x_{-1}, x_{0} \in U\left(x^{*}, r_{2}\right)-\left\{x^{*}\right\}$. Then

$$
\begin{aligned}
\left\|x_{1}-x^{*}\right\| & =\left\|x_{0}-x^{*}-A(F)\left(x_{0}, x_{-1}\right)^{-1} F\left(x_{0}\right)\right\| \\
& =\left\|A(F)\left(x_{0}, x_{-1}\right)^{-1}\left[F\left(x^{*}\right)-F\left(x_{0}\right)-A(F)\left(x_{0}, x_{-1}\right)\left(x^{*}-x_{0}\right)\right]\right\| \\
& \leq \varphi_{2}\left(\left\|x_{0}-x^{*}\right\|,\left\|x_{-1}-x^{*}\right\|\right)\left\|x_{0}-x^{*}\right\|^{p+1} \\
& <\varphi_{3}\left(r_{2}, r_{2}\right) r_{2}^{p}\left\|x_{0}-x^{*}\right\|=\left\|x_{0}-x^{*}\right\|<r_{2},
\end{aligned}
$$


which shows that $x_{1} \in U\left(x^{*}, r_{2}\right)$ and $x_{2}$ is well defined. By a simple inductive argument as in the preceding estimate we get

$$
\begin{aligned}
\left\|x_{k+1}-x^{*}\right\| & =\left\|x_{k}-x^{*}-A(F)\left(x_{k}, x_{k-1}\right)^{-1} F\left(x_{k}\right)\right\| \\
& \leq\left\|A(F)\left(x_{k}, x_{k-1}\right)^{-1}\left[F\left(x^{*}\right)-F\left(x_{k}\right)-A(F)\left(x_{k}, x_{k-1}\right)\left(x^{*}-x_{k}\right)\right]\right\| \\
& \leq \varphi_{2}\left(\left\|x_{k}-x^{*}\right\|,\left\|x_{k-1}-x^{*}\right\|\right)\left\|x_{k}-x^{*}\right\|^{p+1} \\
& <\varphi_{3}\left(r_{2}, r_{2}\right) r_{2}^{p}\left\|x_{k}-x^{*}\right\|=\left\|x_{k}-x^{*}\right\|<r_{2},
\end{aligned}
$$

which shows $\lim _{k \rightarrow \infty} x_{k}=x^{*}$ and $x_{k+1} \in U\left(x^{*}, r_{2}\right)$.

Remark 2.5. (a) Hypothesis (2.3) specializes to Newton-Mysovskiutype if $A(F)(x)=F^{\prime}(x)$ [7, 11, 14]. However, if $F$ is not Fréchet-differentiable, then our results extend the applicability of the iterative algorithm (1.2).

(b) Theorem 2.1 has a practical value although we do not show that $x^{*}$ is a solution of the equation $F(x)=0$, since this may be shown in another way.

(c) Hypothesis (2.13) can be replaced by a stronger one:

$$
\begin{aligned}
\| A(F)(y, x)^{-1}[F(x)-F(z)-A(F) & (y, x)(x-z)] \| \\
& \leq \varphi_{3}(\|z-y\|,\|z-x\|)\|z-y\|^{p+1} .
\end{aligned}
$$

3. Applications to $g$-fractional calculus. Here the background needed comes from [4].

Definition 3.1. Let $\alpha>0,\lceil\alpha\rceil=n(\lceil\cdot\rceil$ is the ceiling of the number). Let $g \in A C([a, b])$ (absolutely continuous functions) be strictly increasing. We assume that $\left(f \circ g^{-1}\right)^{(n)} \circ g \in L_{\infty}([a, b])$, where $f:[a, b] \rightarrow \mathbb{N}$.

We define the left generalized $g$-fractional derivative of $f$ of order $\alpha$ as follows:

$$
\left(D_{a+; g}^{\alpha} f\right)(x):=\frac{1}{\Gamma(n-\alpha)} \int_{a}^{x}(g(x)-g(t))^{n-\alpha-1} g^{\prime}(t)\left(f \circ g^{-1}\right)^{(n)}(g(t)) d t
$$

for $a \leq x \leq b$, where $\Gamma$ is the gamma function.

If $\alpha \notin \mathbb{N}$, we have $D_{a+; g}^{\alpha} f \in C([a, b])$.

We set

$$
\begin{aligned}
& D_{a+; g}^{n} f(x)=\left(\left(f \circ g^{-1}\right)^{(n)} \circ g\right)(x), \\
& D_{a+; g}^{0} f(x)=f(x), \quad \forall x \in[a, b] .
\end{aligned}
$$

If $g=\mathrm{id}$, then

$$
D_{a+; g}^{\alpha} f=D_{a+; \mathrm{id}}^{\alpha} f=D_{* a}^{\alpha} f,
$$

the usual left Caputo fractional derivative. 
We will use the following $g$-left fractional generalized Taylor formula from [4.

TheOrem 3.2. Let $g \in A C([a, b])$ be strictly increasing. Assume that $f \circ g^{-1} \in A C^{n}([g(a), g(b)])$ (this means $\left.\left(f \circ g^{-1}\right)^{(n-1)} \in A C([g(a), g(b)])\right)$, where $\mathbb{N} \ni n=\lceil\alpha\rceil, \alpha>0$. Also, assume that $\left(f \circ g^{-1}\right)^{(n)} \circ g \in L_{\infty}([a, b])$. Then

$$
\begin{aligned}
f(x)-f(a)= & \sum_{k=1}^{n-1} \frac{\left(f \circ g^{-1}\right)^{(k)}(g(a))}{k !}(g(x)-g(a))^{k} \\
& +\frac{1}{\Gamma(\alpha)} \int_{a}^{x}(g(x)-g(t))^{\alpha-1} g^{\prime}(t)\left(D_{a+; g}^{\alpha} f\right)(t) d t
\end{aligned}
$$

for all $x \in[a, b]$. The remainder of (3.1) is a continuous function in $x \in[a, b]$.

Here we are going to operate more generally. We consider $f \in C^{n}([a, b])$. We define the left $g$-fractional derivative of $f$ of order $\alpha$ as follows:

$$
\left(D_{y+; g}^{\alpha} f\right)(x):=\frac{1}{\Gamma(n-\alpha)} \int_{y}^{x}(g(x)-g(t))^{n-\alpha-1} g^{\prime}(t)\left(f \circ g^{-1}\right)^{(n)}(g(t)) d t
$$

for any $a \leq y \leq x \leq b$;

$$
D_{y+; g}^{n} f(x)=\left(\left(f \circ g^{-1}\right)^{(n)} \circ g\right)(x), \quad \forall x, y \in[a, b],
$$

and

$$
D_{y+; g}^{0} f(x)=f(x), \quad \forall x \in[a, b] .
$$

For $\alpha>0, \alpha \notin \mathbb{N}$, by convention we set

$$
\left(D_{y+; g}^{\alpha} f\right)(x)=0, \quad \forall x, y \in[a, b], x<y .
$$

Similarly, we define

$$
\left(D_{x+; g}^{\alpha} f\right)(y):=\frac{1}{\Gamma(n-\alpha)} \int_{x}^{y}(g(y)-g(t))^{n-\alpha-1} g^{\prime}(t)\left(f \circ g^{-1}\right)^{(n)}(g(t)) d t
$$

for any $a \leq x \leq y \leq b$;

$$
D_{x+; g}^{n} f(y)=\left(\left(f \circ g^{-1}\right)^{(n)} \circ g\right)(y), \quad \forall x, y \in[a, b],
$$

and

$$
D_{x+; g}^{0} f(y)=f(y), \quad \forall y \in[a, b] .
$$

For $\alpha>0, \alpha \notin \mathbb{N}$, by convention we set

$$
\left(D_{x+; g}^{\alpha} f\right)(y)=0, \quad \forall x, y \in[a, b], y<x .
$$


Assuming $\left(f \circ g^{-1}\right)^{(n)} \circ g \in L_{\infty}([a, b])$, we get

$$
\begin{aligned}
\left|\left(D_{a+; g}^{\alpha} f\right)(x)\right| & \leq \frac{1}{\Gamma(n-\alpha)} \int_{a}^{x}(g(x)-g(t))^{n-\alpha-1} g^{\prime}(t)\left|\left(f \circ g^{-1}\right)^{(n)}(g(t))\right| d t \\
& \leq \frac{\left\|\left(f \circ g^{-1}\right)^{(n)} \circ g\right\|_{\infty,[a, b]}}{\Gamma(n-\alpha)} \int_{a}^{x}(g(x)-g(t))^{n-\alpha-1} g^{\prime}(t) d t \\
& =\frac{\left\|\left(f \circ g^{-1}\right)^{(n)} \circ g\right\|_{\infty,[a, b]}}{\Gamma(n-\alpha+1)}(g(x)-g(a))^{n-\alpha} \\
& \leq \frac{\left\|\left(f \circ g^{-1}\right)^{(n)} \circ g\right\|_{\infty,[a, b]}}{\Gamma(n-\alpha+1)}(g(b)-g(a))^{n-\alpha}, \quad \forall x \in[a, b] .
\end{aligned}
$$

Hence

$$
\left(D_{a+; g}^{\alpha} f\right)(a)=0
$$

and

$$
\left(D_{y+; g}^{\alpha} f\right)(y)=\left(D_{x+; g}^{\alpha} f\right)(x)=0, \quad \forall x, y \in[a, b]
$$

Thus when $\alpha>0, \alpha \notin \mathbb{N}$, both $D_{y+; g}^{\alpha} f, D_{x+; g}^{\alpha} f \in C([a, b])$.

Notice also that

$$
f \circ g^{-1} \in A C^{n}([g(x), g(b)]) \quad \text { and } \quad\left(f \circ g^{-1}\right)^{(n)} \circ g \in L_{\infty}([x, b]) \text {, }
$$

and of course $g \in A C([x, b])$, and $g$ is strictly increasing over $[x, b]$ for all $x \in[a, b]$.

Hence by Theorem 3.2 we obtain

$$
\begin{aligned}
f(x)-f(y)= & \sum_{k=1}^{n-1} \frac{\left(f \circ g^{-1}\right)^{(k)}(g(y))}{k !}(g(x)-g(y))^{k} \\
& +\frac{1}{\Gamma(\alpha)} \int_{y}^{x}(g(x)-g(t))^{\alpha-1} g^{\prime}(t)\left(D_{y+; g}^{\alpha} f\right)(t) d t, \quad \forall x \in[y, b],
\end{aligned}
$$

and

$$
\begin{aligned}
f(y)-f(x)= & \sum_{k=1}^{n-1} \frac{\left(f \circ g^{-1}\right)^{(k)}(g(x))}{k !}(g(y)-g(x))^{k} \\
& +\frac{1}{\Gamma(\alpha)} \int_{x}^{y}(g(y)-g(t))^{\alpha-1} g^{\prime}(t)\left(D_{x+; g}^{\alpha} f\right)(t) d t, \quad \forall y \in[x, b] .
\end{aligned}
$$


We also define the following linear operator:

$\left(A_{1}(f)\right)(x, y):=$

$\left\{\begin{array}{l}\sum_{k=1}^{n-1} \frac{\left(f \circ g^{-1}\right)^{(k)}(g(y))}{k !}(g(x)-g(y))^{k-1}+\left(D_{y+; g}^{\alpha} f\right)(x) \frac{(g(x)-g(y))^{\alpha-1}}{\Gamma(\alpha+1)} \text { for } x>y, \\ \sum_{k=1}^{n-1} \frac{\left(f \circ g^{-1}\right)^{(k)}(g(x))}{k !}(g(y)-g(x))^{k-1}+\left(D_{x+; g}^{\alpha} f\right)(y) \frac{(g(y)-g(x))^{\alpha-1}}{\Gamma(\alpha+1)} \text { for } x<y, \\ f^{(n)}(x) \text { when } x=y,\end{array}\right.$ for $x, y \in[a, b], \alpha>0, n=\lceil\alpha\rceil$.

We may assume that

$$
\begin{aligned}
\left|\left(A_{1}(f)\right)(x, x)-\left(A_{1}(f)\right)(y, y)\right| & =\left|f^{(n)}(x)-f^{(n)}(y)\right|, \\
\left|\left(f^{(n)} \circ g^{-1}\right)(g(x))-\left(f^{(n)} \circ g^{-1}\right)(g(y))\right| & \leq \Phi|g(x)-g(y)|,
\end{aligned}
$$

for $x, y \in[a, b]$, where $\Phi>0$.

Now we estimate $I_{1}:=\left|f(x)-f(y)-\left(A_{1}(f)\right)(x, y)(g(x)-g(y))\right|$ in two cases. If $x>y$ then

$$
\begin{aligned}
I_{1} & =\mid \frac{1}{\Gamma(\alpha)} \int_{y}^{x}(g(x)-g(t))^{\alpha-1} g^{\prime}(t)\left(D_{y+; g}^{\alpha} f\right)(t) d t \\
& -\left(D_{y+; g}^{\alpha} f\right)(x) \frac{(g(x)-g(y))^{\alpha}}{\Gamma(\alpha+1)} \mid \\
& =\frac{1}{\Gamma(\alpha)}\left|\int_{y}^{x}(g(x)-g(t))^{\alpha-1} g^{\prime}(t)\left(\left(D_{y+; g}^{\alpha} f\right)(t)-\left(D_{y+; g}^{\alpha} f\right)(x)\right) d t\right| \\
& \leq \frac{1}{\Gamma(\alpha)} \int_{y}^{x}(g(x)-g(t))^{\alpha-1} g^{\prime}(t)\left|\left(D_{y+; g}^{\alpha} f\right)(t)-\left(D_{y+; g}^{\alpha} f\right)(x)\right| d t .
\end{aligned}
$$

We can assume that

$$
\left|\left(D_{y+; g}^{\alpha} f\right)(t)-\left(D_{y+; g}^{\alpha} f\right)(x)\right| \leq \lambda_{1}|g(t)-g(x)|
$$

for all $t, x, y \in[a, b]$ with $x \geq t \geq y$, for some $\lambda_{1}>0$. Then

$$
\begin{aligned}
I_{1} & \leq \frac{\lambda_{1}}{\Gamma(\alpha)} \int_{y}^{x}(g(x)-g(t))^{\alpha-1} g^{\prime}(t)(g(x)-g(t)) d t \\
& =\frac{\lambda_{1}}{\Gamma(\alpha)} \int_{y}^{x}(g(x)-g(t))^{\alpha} g^{\prime}(t) d t=\frac{\lambda_{1}}{\Gamma(\alpha)} \frac{(g(x)-g(y))^{\alpha+1}}{\alpha+1} .
\end{aligned}
$$

We have proved that

$$
\begin{aligned}
& \left|f(x)-f(y)-\left(A_{1}(f)\right)(x, y)(g(x)-g(y))\right| \\
& \leq \frac{\lambda_{1}}{\Gamma(\alpha)} \frac{(g(x)-g(y))^{\alpha+1}}{\alpha+1}, \quad \forall x, y \in[a, b], x>y .
\end{aligned}
$$


Now we assume that $y>x$. Then

$$
\begin{aligned}
I_{1} & =\left|f(y)-f(x)-\left(A_{1}(f)\right)(x, y)(g(y)-g(x))\right| \\
& =\mid \frac{1}{\Gamma(\alpha)} \int_{x}^{y}(g(y)-g(t))^{\alpha-1} g^{\prime}(t)\left(D_{x+; g}^{\alpha} f\right)(t) d t \\
& \quad-\left(D_{x+; g}^{\alpha} f\right)(y) \frac{(g(y)-g(x))^{\alpha}}{\Gamma(\alpha+1)} \mid \\
& =\frac{1}{\Gamma(\alpha)}\left|\int_{x}^{y}(g(y)-g(t))^{\alpha-1} g^{\prime}(t)\left(\left(D_{x+; g}^{\alpha} f\right)(t)-\left(D_{x+; g}^{\alpha} f\right)(y)\right) d t\right| \\
& \leq \frac{1}{\Gamma(\alpha)} \int_{x}^{y}(g(y)-g(t))^{\alpha-1} g^{\prime}(t)\left|\left(D_{x+; g}^{\alpha} f\right)(t)-\left(D_{x+; g}^{\alpha} f\right)(y)\right| d t .
\end{aligned}
$$

We can assume that

$$
\left|\left(D_{x+; g}^{\alpha} f\right)(t)-\left(D_{x+; g}^{\alpha} f\right)(y)\right| \leq \lambda_{2}|g(t)-g(y)|
$$

for all $t, y, x \in[a, b]$ with $y \geq t \geq x$, for some $\lambda_{2}>0$. Then

$$
\begin{aligned}
I_{1} & \leq \frac{\lambda_{2}}{\Gamma(\alpha)} \int_{x}^{y}(g(y)-g(t))^{\alpha-1} g^{\prime}(t)(g(y)-g(t)) d t \\
& =\frac{\lambda_{2}}{\Gamma(\alpha)} \int_{x}^{y}(g(y)-g(t))^{\alpha} g^{\prime}(t) d t=\frac{\lambda_{2}}{\Gamma(\alpha)} \frac{(g(y)-g(x))^{\alpha+1}}{\alpha+1} .
\end{aligned}
$$

We have proved that

$$
\begin{aligned}
& \left|f(x)-f(y)-\left(A_{1}(f)\right)(x, y)(g(x)-g(y))\right| \\
& \leq \frac{\lambda_{2}}{\Gamma(\alpha)} \frac{(g(y)-g(x))^{\alpha+1}}{\alpha+1}, \quad \forall x, y \in[a, b], y>x .
\end{aligned}
$$

Conclusion 3.3. Set $\lambda:=\max \left(\lambda_{1}, \lambda_{2}\right)$. Then

$$
\begin{aligned}
\mid f(x)-f(y)-\left(A_{1}(f)\right) & (x, y)(g(x)-g(y)) \mid \\
\leq & \frac{\lambda}{\Gamma(\alpha)} \frac{|g(x)-g(y)|^{\alpha+1}}{\alpha+1}, \quad \forall x, y \in[a, b] .
\end{aligned}
$$

Notice that $(3.3)$ is trivially true when $x=y$.

One may assume that

$$
\lambda / \Gamma(\alpha)<1 .
$$

Now based on (3.2) and (3.3), we can apply our numerical methods presented in Section 2 to solve $f(x)=0$.

Definition 3.4. Let $\alpha>0,\lceil\alpha\rceil=n$. Let $g \in A C([a, b])$ be strictly increasing. Assume that $\left(f \circ g^{-1}\right)^{(n)} \circ g \in L_{\infty}([a, b])$, where $f:[a, b] \rightarrow \mathbb{R}$. 
We define the right generalized $g$-fractional derivative of $f$ of order $\alpha$ as follows:

$$
\left(D_{b-; g}^{\alpha} f\right)(x):=\frac{(-1)^{n}}{\Gamma(n-\alpha)} \int_{x}^{b}(g(t)-g(x))^{n-\alpha-1} g^{\prime}(t)\left(f \circ g^{-1}\right)^{(n)}(g(t)) d t
$$

for $x \in[a, b]$.

If $\alpha \notin \mathbb{N}$, we have $D_{b-; g}^{\alpha} f \in C([a, b])$.

We set

$$
\begin{aligned}
& D_{b-; g}^{n} f(x)=(-1)^{n}\left(\left(f \circ g^{-1}\right)^{(n)} \circ g\right)(x), \\
& D_{b-; g}^{0} f(x)=f(x), \quad \forall x \in[a, b] .
\end{aligned}
$$

If $g=\mathrm{id}$, then

$$
D_{b-; g}^{\alpha} f=D_{b-; \mathrm{id}}^{\alpha} f=D_{b-}^{\alpha} f,
$$

the usual right Caputo fractional derivative.

We will use the following $g$-right fractional generalized Taylor formula from [4].

Theorem 3.5. Let $g \in A C([a, b])$ be strictly increasing. Assume that $f \circ g^{-1} \in A C^{n}([g(a), g(b)])$, where $\mathbb{N} \ni n=\lceil\alpha\rceil, \alpha>0$. Also assume that $\left(f \circ g^{-1}\right)^{(n)} \circ g \in L_{\infty}([a, b])$. Then

$$
\begin{aligned}
f(x)-f(b)= & \sum_{k=1}^{n-1} \frac{\left(f \circ g^{-1}\right)^{(k)}(g(b))}{k !}(g(x)-g(b))^{k} \\
& +\frac{1}{\Gamma(\alpha)} \int_{x}^{b}(g(t)-g(x))^{\alpha-1} g^{\prime}(t)\left(D_{b-; g}^{\alpha} f\right)(t) d t
\end{aligned}
$$

for all $a \leq x \leq b$. The remainder of (3.5) is a continuous function in $x \in[a, b]$.

Here we are going to operate more generally. We consider $f \in C^{n}([a, b])$. We define the right $g$-fractional derivative of $f$ of order $\alpha$ as follows:

$$
\left(D_{y-; g}^{\alpha} f\right)(x)=\frac{(-1)^{n}}{\Gamma(n-\alpha)} \int_{x}^{y}(g(t)-g(x))^{n-\alpha-1} g^{\prime}(t)\left(f \circ g^{-1}\right)^{(n)}(g(t)) d t
$$

for $x \in[a, y]$, where $y \in[a, b]$;

$$
\begin{aligned}
& \left(D_{y-; g}^{n} f\right)(x)=(-1)^{n}\left(\left(f \circ g^{-1}\right)^{(n)} \circ g\right)(x), \quad \forall x, y \in[a, b], \\
& \left(D_{y-; g}^{0} f\right)(x)=f(x), \quad \forall x \in[a, b] .
\end{aligned}
$$

For $\alpha>0, \alpha \notin \mathbb{N}$, by convention we set

$$
\left(D_{y-; g}^{\alpha} f\right)(x)=0, \quad \forall x, y \in[a, b], x>y .
$$


Similarly, we define

$$
\left(D_{x-; g}^{\alpha} f\right)(y)=\frac{(-1)^{n}}{\Gamma(n-\alpha)} \int_{y}^{x}(g(t)-g(y))^{n-\alpha-1} g^{\prime}(t)\left(f \circ g^{-1}\right)^{(n)}(g(t)) d t
$$

for $y \in[a, x]$, where $x \in[a, b]$, and

$$
\begin{aligned}
& \left(D_{x-; g}^{n} f\right)(y)=(-1)^{n}\left(\left(f \circ g^{-1}\right)^{(n)} \circ g\right)(y), \quad \forall x, y \in[a, b], \\
& \left(D_{x-; g}^{0} f\right)(y)=f(y), \quad \forall y \in[a, b] .
\end{aligned}
$$

For $\alpha>0, \alpha \notin \mathbb{N}$, by convention we set

$$
\left(D_{x-; g}^{\alpha} f\right)(y)=0, \quad \forall x, y \in[a, b], y>x .
$$

Assuming $\left(f \circ g^{-1}\right)^{(n)} \circ g \in L_{\infty}([a, b])$, we get

$$
\begin{aligned}
\left|\left(D_{b-; g}^{\alpha} f\right)(x)\right| & \leq \frac{\left\|\left(f \circ g^{-1}\right)^{(n)} \circ g\right\|_{\infty,[a, b]}}{\Gamma(n-\alpha+1)}(g(b)-g(x))^{n-\alpha} \\
& \leq \frac{\left\|\left(f \circ g^{-1}\right)^{(n)} \circ g\right\|_{\infty,[a, b]}}{\Gamma(n-\alpha+1)}(g(b)-g(a))^{n-\alpha}, \quad \forall x \in[a, b] .
\end{aligned}
$$

That is,

$$
\left(D_{b-; g}^{\alpha} f\right)(b)=0
$$

and

$$
\left(D_{y-; g}^{\alpha} f\right)(y)=\left(D_{x-; g}^{\alpha} f\right)(x)=0, \quad \forall x, y \in[a, b] .
$$

Thus when $\alpha>0, \alpha \notin \mathbb{N}$, both $D_{y-; g}^{\alpha} f, D_{x-; g}^{\alpha} f \in C([a, b])$.

Notice also that $f \circ g^{-1} \in A C^{n}([g(a), g(x)])$ and $\left(f \circ g^{-1}\right)^{(n)} \circ g \in$ $L_{\infty}([a, x])$, and of course $g \in A C([a, x])$, and $g$ is strictly increasing over $[a, x]$ for all $x \in[a, b]$.

Hence by Theorem 3.5 we obtain

$$
\begin{aligned}
f(x)-f(y) & =\sum_{k=1}^{n-1} \frac{\left(f \circ g^{-1}\right)^{(k)}(g(y))}{k !}(g(x)-g(y))^{k} \\
& +\frac{1}{\Gamma(\alpha)} \int_{x}^{y}(g(t)-g(x))^{\alpha-1} g^{\prime}(t)\left(D_{y-; g}^{\alpha} f\right)(t) d t, \quad \forall a \leq x \leq y \leq b .
\end{aligned}
$$

Also, we have

$$
\begin{aligned}
f(y)-f(x) & =\sum_{k=1}^{n-1} \frac{\left(f \circ g^{-1}\right)^{(k)}(g(x))}{k !}(g(y)-g(x))^{k} \\
& +\frac{1}{\Gamma(\alpha)} \int_{y}^{x}(g(t)-g(y))^{\alpha-1} g^{\prime}(t)\left(D_{x-; g}^{\alpha} f\right)(t) d t, \quad \forall a \leq y \leq x \leq b .
\end{aligned}
$$


We also define the following linear operator:

$\left(A_{2}(f)\right)(x, y):=$

$$
\begin{cases}\sum_{k=1}^{n-1} \frac{\left(f \circ g^{-1}\right)^{(k)}(g(y))}{k !}(g(x)-g(y))^{k-1}-\left(D_{y-; g}^{\alpha} f\right)(x) \frac{(g(y)-g(x))^{\alpha-1}}{\Gamma(\alpha+1)} & \text { for } x<y, \\ \sum_{k=1}^{n-1} \frac{\left(f \circ g^{-1}\right)^{(k)}(g(x))}{k !}(g(y)-g(x))^{k-1}-\left(D_{x-; g}^{\alpha} f\right)(y) \frac{(g(x)-g(y))^{\alpha-1}}{\Gamma(\alpha+1)} & \text { for } x>y, \\ f^{(n)}(x) \text { when } x=y & \end{cases}
$$

for $x, y \in[a, b], \alpha>0, n=\lceil\alpha\rceil$.

We may assume that

$$
\begin{aligned}
\left|\left(A_{2}(f)\right)(x, x)-\left(A_{2}(f)\right)(y, y)\right| & =\left|f^{(n)}(x)-f^{(n)}(y)\right| \\
& \leq \Phi^{*}|g(x)-g(y)|, \quad \forall x, y \in[a, b],
\end{aligned}
$$

where $\Phi^{*}>0$.

Again we estimate, first for $x<y$,

$$
\begin{aligned}
I_{2} & :=\left|f(x)-f(y)-\left(A_{2}(f)\right)(x, y)(g(x)-g(y))\right| \\
& =\mid \frac{1}{\Gamma(\alpha)} \int_{x}^{y}(g(t)-g(x))^{\alpha-1} g^{\prime}(t)\left(D_{y-; g}^{\alpha} f\right)(t) d t \\
& -\left(D_{y-; g}^{\alpha} f\right)(x) \frac{(g(y)-g(x))^{\alpha}}{\Gamma(\alpha+1)} \mid \\
& =\left.\frac{1}{\Gamma(\alpha)}\right|_{x} ^{y}(g(t)-g(x))^{\alpha-1} g^{\prime}(t)\left(\left(D_{y-; g}^{\alpha} f\right)(t)-\left(D_{y-; g}^{\alpha} f\right)(x)\right) d t \mid \\
& \leq \frac{1}{\Gamma(\alpha)} \int_{x}^{y}(g(t)-g(x))^{\alpha-1} g^{\prime}(t)\left|\left(D_{y-; g}^{\alpha} f\right)(t)-\left(D_{y-; g}^{\alpha} f\right)(x)\right| d t .
\end{aligned}
$$

We can assume that

$$
\left|\left(D_{y-; g}^{\alpha} f\right)(t)-\left(D_{y-; g}^{\alpha} f\right)(x)\right| \leq \rho_{1}|g(t)-g(x)|
$$

for all $t, x, y \in[a, b]$ with $y \geq t \geq x$, for some $\rho_{1}>0$. Then

$$
\begin{aligned}
I_{2} & \leq \frac{\rho_{1}}{\Gamma(\alpha)} \int_{x}^{y}(g(t)-g(x))^{\alpha-1} g^{\prime}(t)(g(t)-g(x)) d t \\
& =\frac{\rho_{1}}{\Gamma(\alpha)} \int_{x}^{y}(g(t)-g(x))^{\alpha} g^{\prime}(t) d t \\
& =\frac{\rho_{1}}{\Gamma(\alpha)} \frac{(g(y)-g(x))^{\alpha+1}}{\alpha+1} .
\end{aligned}
$$


We have proved that

$$
\begin{aligned}
& \left|f(x)-f(y)-\left(A_{2}(f)\right)(x, y)(g(x)-g(y))\right| \\
& \leq \frac{\rho_{1}}{\Gamma(\alpha)} \frac{(g(y)-g(x))^{\alpha+1}}{\alpha+1}, \quad \forall x, y \in[a, b], x<y .
\end{aligned}
$$

Now assume that $x>y$. Then

$$
\begin{aligned}
I_{2} & =\left|f(y)-f(x)-\left(A_{2}(f)\right)(x, y)(g(y)-g(x))\right| \\
& =\left|f(y)-f(x)+\left(A_{2}(f)\right)(x, y)(g(x)-g(y))\right| \\
& =\mid \frac{1}{\Gamma(\alpha)} \int_{y}^{x}(g(t)-g(y))^{\alpha-1} g^{\prime}(t)\left(D_{x-; g}^{\alpha} f\right)(t) d t \\
& \quad-\left(D_{x-; g}^{\alpha} f\right)(y) \frac{(g(x)-g(y))^{\alpha}}{\Gamma(\alpha+1)} \mid \\
& =\frac{1}{\Gamma(\alpha)} \int_{y}^{x}(g(t)-g(y))^{\alpha-1} g^{\prime}(t)\left(\left(D_{x-; g}^{\alpha} f\right)(t)-\left(D_{x-; g}^{\alpha} f\right)(y)\right) d t \mid \\
& \leq \frac{1}{\Gamma(\alpha)} \int_{y}^{x}(g(t)-g(y))^{\alpha-1} g^{\prime}(t)\left|\left(D_{x-; g}^{\alpha} f\right)(t)-\left(D_{x-; g}^{\alpha} f\right)(y)\right| d t .
\end{aligned}
$$

We can assume that

$$
\left|\left(D_{x-; g}^{\alpha} f\right)(t)-\left(D_{x-; g}^{\alpha} f\right)(y)\right| \leq \rho_{2}|g(t)-g(y)|
$$

for all $t, y, x \in[a, b]$ with $x \geq t \geq y$, for some $\rho_{2}>0$. Then

$$
\begin{aligned}
I_{2} & \leq \frac{\rho_{2}}{\Gamma(\alpha)} \int_{y}^{x}(g(t)-g(y))^{\alpha-1} g^{\prime}(t)(g(t)-g(y)) d t \\
& =\frac{\rho_{2}}{\Gamma(\alpha)} \int_{y}^{x}(g(t)-g(y))^{\alpha} g^{\prime}(t) d t=\frac{\rho_{2}}{\Gamma(\alpha)} \frac{(g(x)-g(y))^{\alpha+1}}{\alpha+1} .
\end{aligned}
$$

We have proved that

$$
\begin{aligned}
& \left|f(x)-f(y)-\left(A_{2}(f)\right)(x, y)(g(x)-g(y))\right| \\
& \leq \frac{\rho_{2}}{\Gamma(\alpha)} \frac{(g(x)-g(y))^{\alpha+1}}{\alpha+1}, \quad \forall x, y \in[a, b], x>y .
\end{aligned}
$$

Conclusion 3.6. Set $\rho:=\max \left(\rho_{1}, \rho_{2}\right)$. Then

$$
\begin{aligned}
\mid f(x)-f(y)-\left(A_{2}(f)\right) & (x, y)(g(x)-g(y)) \mid \\
\leq & \frac{\rho}{\Gamma(\alpha)} \frac{|g(x)-g(y)|^{\alpha+1}}{\alpha+1}, \quad \forall x, y \in[a, b] .
\end{aligned}
$$

Notice that $(3.7)$ is trivially true when $x=y$. 
One may assume that

$$
\frac{\rho}{\Gamma(\alpha)}<1
$$

Now based on (3.6) and (3.7), we can apply our numerical methods presented in this article to solve $f(x)=0$.

In both fractional applications $\alpha+1 \geq 2$, that is, $\alpha \geq 1$.

Also some examples for $g$ follow:

$$
\begin{aligned}
& g(x)=e^{x}, \quad x \in[a, b] \subset \mathbb{R}, \\
& g(x)=\sin x, \\
& g(x)=\tan x, \quad x \in[-\pi / 2+\varepsilon, \pi / 2-\varepsilon], \text { where } \varepsilon>0 \text { is small. }
\end{aligned}
$$

Indeed, the above examples are strictly increasing and absolutely continuous functions.

Remark 3.7. (a) Returning to Conclusion 3.3, we see that Proposition 2.2 can be applied if $p=\alpha, g(t)=t, F(t)=f(t), A(F)(s, t)=$ $A_{1}(f)(s, t)$ and

$$
\varphi_{1}(s, t)=\frac{\lambda|s-t|^{p}}{(\alpha+1) \Gamma(\alpha) \mu}
$$

for each $s, t \in[a, b]$.

(b) According to Conclusion 3.6, we can again use Proposition 2.2 as in (a) but we must choose $A(F)(s, t)=A_{2}(f)(s, t)$ and

for each $s, t \in[a, b]$.

$$
\varphi_{1}(s, t)=\frac{\rho|s-t|^{p}}{(\alpha+1) \Gamma(\alpha) \mu}
$$

\section{References}

[1] S. Amat and S. Busquier, Third-order iterative methods under Kantorovich conditions, J. Math. Anal. Appl. 336 (2007), 243-261.

[2] S. Amat, S. Busquier and S. Plaza, Chaotic dynamics of a third-order Newton-type method, J. Math. Anal. Appl. 366 (2010), 164-174.

[3] G. Anastassiou, Fractional representation formulae and right fractional inequalities, Math. Computer Modelling 54 (2011), 3098-3115.

[4] G. Anastassiou, Advanced fractional Taylor's formulae, J. Comput. Anal. Appl., to appear (2016).

[5] I. K. Argyros, Newton-like methods in partially ordered linear spaces, J. Approx. Theory Appl. 9 (1993), 1-10.

[6] I. K. Argyros, Results on controlling the residuals of perturbed Newton-like methods on Banach spaces with a convergence structure, Southwest J. Pure Appl. Math. 1 (1995), 32-38.

[7] I. K. Argyros, Convergence and Applications of Newton-Type Iterations, Springer, New York, 2008. 
[8] K. Diethelm, The Analysis of Fractional Differential Equations, Lecture Notes in Math. 2004, Springer, New York, 2010.

[9] J. A. Ezquerro, J. M. Gutiérrez, M. Á. Hernández, N. Romero and M. J. Rubio, The Newton method: From Newton to Kantorovich, Gac. R. Soc. Mat. Esp. 13 (2010), 53-76 (in Spanish).

[10] J. A. Ezquerro and M. Á. Hernández, Newton-type methods of high order and domains of semilocal and global convergence, Appl. Math. Comput. 214 (2009), 142154.

[11] L. V. Kantorovich and G. P. Akilov, Functional Analysis in Normed Spaces, Pergamon Press, New York, 1964.

[12] Á. A. Magreñán, Different anomalies in a Jarratt family of iterative root finding methods, Appl. Math. Comput. 233 (2014), 29-38.

[13] Á. A. Magreñán, A new tool to study real dynamics: The convergence plane, Appl. Math. Comput. 248 (2014), 215-224.

[14] F. A. Potra and V. Pták, Nondiscrete Induction and Iterative Processes, Pitman Publ., London, 1984.

[15] P. D. Proinov, New general convergence theory for iterative processes and its applications to Newton-Kantorovich type theorems, J. Complexity 26 (2010), 3-42.

George A. Anastassiou

Department of Mathematical Sciences

University of Memphis

Memphis, TN 38152, U.S.A.

E-mail: ganastss@memphis.edu
Ioannis K. Argyros Department of Mathematical Sciences Cameron University Lawton, OK 73505, U.S.A. E-mail: iargyros@cameron.edu 